\title{
A LYAPUNOV-TYPE INEQUALITY FOR A TWO-TERM EVEN-ORDER DIFFERENTIAL EQUATION
}

\author{
Xiading Yang, Yong-In Kim and Kueiming Lo
}

\begin{abstract}
In this note, a new inequality is obtained for a two-term even-order linear differential equation, which generalizes the well-known Lyapunov-type inequality for second order linear differential equations.
\end{abstract}

Mathematics subject classification (2010): 34A40.

Keywords and phrases: Lyapunov-type inequality, even-order differential equation, Green function.

\section{REFERENCES}

[1] S. S. CHENG, Lyapunov inequalities for differential and difference equations, Fasc. Math. 23 (1991), $25-41$.

[2] O. Dosly, P. RehaK, Half-linear Differential Equations, Math. Stud., vol 202, North-Holland, 2005.

[3] S. B. Eliason, A Lyapunov type inequality for certain nonlinear differential equation, J. London Math. Soc. 3 (1970), 461-466.

[4] G. Guseinov, B. Kaymakcalan, Lyapunov inequalities for discrete linear Hamiltonian system, Comput. Math. Appl. 45 (2003), 1399-1416.

[5] P. Hartmann, Ordinary Differential Equations, second ed., Birkhauser, Boston, 1982.

[6] H. Hochstadt, On an inequality of Lyapunov, Proc. Amer. Math. Soc. 22 (1969), 282-284.

[7] C. Lee, C. Yeh, C. Hong, R. P. Agarwal, Lyapunov and Wirtinger inequalities, Appl. Math. Lett. 17 (2004), 847-853.

[8] W. Leighton, On Lyapunov's inequality, Proc. Amer. Math. Soc. 33 (1972), 627-628.

[9] Z. NeHARI, On an inequality of Lyapunov, In "Studies in Mathematical Analysis and Related Topics", pp. 256-261, Stanford Univ. Press, Stanford, CA, 1962.

[10] B. G. PAChPATTE, On Lyapunov-type inequalities for certain higher order differential equations, J. Math. Anal. Appl. 195 (1995), 527-536.

[11] N. PARHI, S. PANIGRAhI, On Liapunov-type inequality for third-order differential equations, J. Math. Anal. Appl. 233 (1999), 445-464.

[12] William T. ReID, Interrelations between a Trace Formula and Lyapunov Type Inequalities, J. Differential Equations 23 (1977), 448-458.

[13] A. TIRYAKI, M. UnAL AND D. CAKMAK, Lyapunov-type inequalities for nonlinear systems, J. Math. Anal. Appl. 332 (2007), 497-511.

[14] X. YANG, On Lyapunov-type inequalities for certain higher-order differential equations, Appl. Math. Computation, 134 (2003), 307-317. 